\title{
Multidrug resistance-associated protein 3 and Bcl-2 contribute to multidrug resistance by vinorelbine in lung adenocarcinoma
}

\author{
XUAN HONG ${ }^{1}$, ZHAO-YANG YANG ${ }^{1}$, MENG WANG $^{1}$, LU LU $^{2}$, YING-HONG LI ${ }^{1}$, \\ XISHAN $\mathrm{HAO}^{3}$ and GONG-YAN $\mathrm{CHEN}^{1}$ \\ ${ }^{1}$ Department of Medical Oncology, The Third Affiliated Hospital of Harbin Medical University; ${ }^{2}$ Department of Medical \\ Oncology, The Fourth Affiliated Hospital of Harbin Medical University; ${ }^{3}$ Department of General Surgery, \\ The Tumor Hospital of Tianjin Medical University, P.R. China
}

Received May 23, 2011; Accepted July 4, 2011

DOI: $10.3892 /$ ijmm.2011.779

\begin{abstract}
Although cancer cells initially respond to vinorelbine (NVB), the acquisition of resistance to the treatment is the main cause of chemotherapeutic failure in lung cancer. The intrinsic mechanism of drug resistance induced by NVB in lung cancer is not clear and tumor cell models to study NVB resistance have not been widely studied. We previously established a NVB resistant cell line, Anip973/NVB, derived from the Anip973 lung adenocarcinoma cell line. The aim of this study was to investigate the molecular mechanisms involved in the resistance to NVB in lung adenocarcinoma. Genetic profiles of Anip973/NVB and Anip973 cells were compared by microarray analysis and qRT-PCR. Tumor xenografts were obtained by grafting Anip973/NVB and Anip973 cells into nude mice and the xenograft response to NVB or control treatment was evaluated. Morphological assessment of xenograft tissues was performed by transmission electron microscopy (TEM). Immunohistochemistry (IHC) was used to compare Bcl-2 and MRP3 protein expression in xenografts. Fifty-five up-regulated genes and 88 down-regulated genes in Anip973/ NVB cells compared with Anip973 cells were identified by cDNA microarray analysis. Up-regulation of MRP3 and Bcl-2 was confirmed by qRT-PCR. NVB inhibits xenografts of Anip973 growth but did not affect xenografts of Anip973/NVB growth. Ultrastructural changes observed by TEM showed that NVB induces apoptosis in the Anip973-treated group but not in the Anip973/NVB-treated group. Higher expression rates of Bcl-2 and MRP3 were observed in Anip973/NVB xenograft cells compared with Anip973 xenograft cells by IHC. In conclusion, in the present study, we identified a set of genes responsible for multidrug resistance in Anip973/NVB cells.
\end{abstract}

Correspondence to: Dr Gong-Yan Chen, Department of Medical Oncology, The Third Affiliated Hospital of Harbin Medical University, Haping Road 150, Harbin 150081, P.R. China

E-mail: chengongyan@medmail.com.cn

Key words: vinorelbine, multidrug resistance, lung adenocarcinoma, MRP3, Bcl-2
Among them, MRP3 and Bcl-2 may participate in lung adenocarcinoma multidrug resistance induced by NVB.

\section{Introduction}

Vinorelbine (Navelbine, NVB, 3,4'-didehydro-4'deoxy- $C^{\prime}$ vinorelbine) is used for chemotherapy of malignancy since the 1990s, and it is chemically different from vinblastine by substitutions on the catharantine moiety of the molecule and is found to bind to the membrane vesicles more intensively than vincristine $(1,2)$. NVB is one of first-line chemotherapeutic drugs for non-small cell lung cancer (NSCLC) patients. The effective rates of the anticancer agent for NSCLC were reported to be between $14-33 \%$ and up to about $50 \%$ in combination chemotherapy with cisplatin, and it is regarded as one of the most effective drugs for NSCLC (3-5). However, although the tumors initially respond to NVB, the acquisition of NVB resistance used in chemotherapy is still the main cause of treatment failure in malignancy. Similarly with cisplatin, resistance of lung cancer cells to NVB is usually accompanied by resistance to other drugs with different structures and cellular targets $(6,7)$. Very little is known about the intrinsic mechanism for inducing multidrug resistance by NVB until now. Identifying the molecular detail leading to acquired multidrug resistance to NVB may be important in developing more effective therapies.

The intrinsic mechanism of drug resistance induced by NVB is not clear and tumor cell models to study NVB resistance are rare until now. We thus established an NVB-resistant cell line from the lung adenocarcinoma Anip973 cell line and named it Anip973/NVB in a previous study (8). The sensitivities of Anip973/NVB cells to various chemotherapeutics were determined by MTT assay. Our previous study showed that the resistance index of the Anip973/NVB cells to NVB was 21.81, and Anip973/NVB cells had multidrug resistant characteristic to several drugs, such as cisplatin, paclitaxel, etoposide, isofosfamide, fluorouracil, dacarbazine, pharmorubicin, with resistance indices of 11.89, 11.68, 6.12, 3.95, 2.86, 2.64 and 2.15 , respectively. Nevertheless, the cells still remained sensitive to irinotecan and gemcitabine.

In order to study the mechanism on multidrug resistance by NVB in lung adenocarcinoma, we investigated the gene expression variance between the Anip973/NVB and Anip973 
cell lines by DNA microarray and qRT-PCR. Then xenografts of Anip973/NVB and Anip973 cells were established to investigate selected drug-resistance proteins.

\section{Materials and methods}

Cell lines. The human lung adenocarcinoma cell line, Anip973, was kindly provided by the Tumor Research Institute of Harbin Medical University. The Anip973/NVB cell line was established by slowly increasing the concentration of NVB (Laboratories Pierre Fabre, France) and its biological characteristics were confirmed (8). Anip973 and Anip973/NVB cells were cultured in RPMI-1640 (HyClone, USA) medium supplemented with $10 \%$ fetal bovine serum, penicillin $(100 \mathrm{U} / \mathrm{ml})$ and streptomycin $(100 \mu \mathrm{g} / \mathrm{ml})$ (Invitrogen, Gaithersburg, MD, USA) in standard culture condition $\left(95 \%\right.$ air, $\left.5 \% \mathrm{CO}_{2}, 37^{\circ} \mathrm{C}\right)$.

Microarray assays and analysis. Human gene oligonucleotide arrays (CapitalBio, China) which contained 7-mer length DNA from 21571 genes was used. Total-RNA was isolated from Anip973 and Anip973/NVB cells using the TRIzol reagent (Invitrogen) according to manufacturer's instructions. Integrity of the RNA was determined by spectrophotometry and electrophoresis. Total-RNA was reverse transcribed into cDNA by the cDNA synthesis kit (Promega, USA). Cy3-dUTP or Cy5-dUTP (Amersham Pharmacia Biotech, USA) was incorporated during reverse transcription of $10 \mu \mathrm{g}$ of total-RNA and primed by $\mathrm{T}_{7}$-Oligo(dt)15 primer (Boya, China). Different fluorescentlabeled probes from Anip973 and Anip973/NVB were mixed. After incubation at $42^{\circ} \mathrm{C}$ overnight in a humidified environment, fluorescent images of hybridized microarrays were scanned with LuxScan 10KA dual channel laser scanner (CapitalBio) using GenePix Pro 4.0 image analysis software (Axon Instruments, USA) according to the manufacturer's instructions. To control labeling differences, the experiment was carried out three times, in which the fluorescent dyes were switched during cDNA synthesis for each cell line. The two ratios were then multiplied to generate the ratio of Anip973/NVB to Anip973 cells, and one gene was considered to be significantly differentially expressed if its ratio was $>2$ in all three experiments.

Quantitative reverse transcription-PCR. Total-RNA was isolated from Anip973 and Anip973/NVB cells using the TRIzol reagent (Invitrogen). cDNA was synthesized from isolated RNA using the RevertAid ${ }^{\mathrm{TM}}$ First Strand cDNA Synthesis kit (MBI, USA) according to manufacturer's instructions. The expression levels of MRP1, MRP3, MDR1, Bcl-2 were validated by real-time quantitative reverse transcription PCR (qRT-PCR). The SYBR-Green master mix from Applied Biosystems was used. qRT-PCR was performed using the ABI PRISM 7000 Sequence Detection system (Applied Biosystems). The $C_{T}$ value of mRNA expression for each sample were normalized with the internal housekeeping gene $\beta$-actin and relative quantitation values were plotted.

The primer sequences of each gene for real-time qPCR were as follows: MDR1, forward, 5'-ATTTCTATAGGTGCT-3' and reverse, 5'-CCGTAGAAACCTTAC-3'; MRP1, forward, 5'-GGACCTGGACTTCGTTCTCA-3' and reverse, 5'-CGTCC AGACTTCTTCATCCG-3'; MRP3, forward, 5'-GCACCAT TGTCGTGGCTACA-3' and reverse, 5'-GCAGGACACCCAG
GACCAT-3'; Bcl-2, forward, 5'-TGCACCTGACGCCCTT CAC-3' and reverse, 5'-AGACAGCCAGGAGAAATCAAAC AG-3'; $\beta$-actin, forward, 5'-GCTGGAAGGTGGACAGCGA-3' and reverse, 5'-GGCATCGTGATGGACTCCG-3'.

Establishment of tumor xenografts. All studies were approved by the institutional animal care and use committee, and they were carried out in accordance with institutional guidelines for animal care. The female BALB/C nude mice were provided by the Beijing Animal Research Institute. The animals were 4-6-weeks-old and weighed 17-21 grams on the day of tumor implantation. Each nude mouse was inoculated with $1 \mathrm{ml}$ of $1 \times 10^{7}$ cells subcutaneously via the left armpit. The 12 models were established with Anip973 cells and the other 12 models were established with the Anip973/NVB cells. When tumors grew to $5-10 \mathrm{~mm}$ in diameter, the animals were grouped and then managed.

The 2 xenograft models were equally assigned to 2 treatment groups respectively: 6 mice for Anip973 cells and 6 mice for Anip973/NVB cells were treated with $0.2 \mathrm{ml}$ of $20 \mathrm{mg} / \mathrm{kg}$ NVB via the vena caudalis (treated groups) and 6 mice for Anip973 cells and 6 mice for Anip973/NVB cells were treated with $0.2 \mathrm{ml}$ of saline in the same way (untreated groups). After 7 days, the mice were sacrificed and the tumors were excised and tumor volumes were measured. The size of the tumors was determined by measuring length (the longest diameter) and width (the shortest diameter). The tumor volume was calculated using the formula: volume $=$ width $^{2} \mathrm{x}$ length $\mathrm{x} 0.52$. The tumor growth inhibition rate (IR) was calculated according to the volume of the tumor: IR $(\%)=(1$-volume of treated group/ volume of untreated group) $\mathrm{x} 100 \%$.

Morphological assessment of apoptosis of xenograft tissues. After the mice were sacrificed, the xenograft tissues were excised and cut into small pieces (1x1 mm), fixed with $2.5 \%$ glutaraldehyde and phosphate buffer solution for $3 \mathrm{~h}$ and washed with $0.1 \mathrm{M}$ sodium phosphate buffer, post-fixed with $1 \%$ osmium tetroxide, dehydrated in graded ethanol and acetonum, embedded in Epon 812 mixture and cut into sections with $50 \mathrm{~nm}$ thickness using an ultramicrotome (Leica, China). After staining with uranyl acetate and lead citrate, the sections were examined by transmission electron microscopy (TEM) (Hitachi-500, Japan).

Immunohistochemical analysis. Excised tumors were embedded in formalin-fixed paraffin and then sectioned into $4 \mu \mathrm{m}$ thick sections, and mounted on glass slides. After deparaffinization, the tissue sections were heated at $120^{\circ} \mathrm{C}$ for $15 \mathrm{~min}$ in $10 \mathrm{mM}$ Tris- $\mathrm{HCl}$ with $1 \mathrm{mM}$ EDTA (pH 9.0). Endogenous peroxidase was blocked with $3 \%$ hydrogen peroxide in methanol for $10 \mathrm{~min}$ at room temperature. The sections were incubated overnight at $4^{\circ} \mathrm{C}$ with antibodies against Bcl-2 (Santa Cruz Biotechnology, Santa Cruz, CA, USA) at a 1:200 dilution and MRP3 (Santa Cruz Biotechnology) at a 1:300 dilution. Then biotinylated immunoglobulin and streptavidin conjugated to peroxidase were added. Finally, 3,3'-diaminobenzidine was added for color development, and hematoxylin was used for counterstaining. Negative controls processed without the primary antibody were performed in each experiment. The mean percentage of positive tumor cells was determined in at least five areas at $\mathrm{x} 400$ 
Table I. The 143 genes up-regulated and down-regulated between Anip973 and Anip973/NVB cells by microarray analysis.

\begin{tabular}{|c|c|c|c|}
\hline Accession no. & Gene name & Gene description & Ratio \\
\hline NM003726 & SCAP1 & Src family associated phosphoprotein 1 & 30.6889 \\
\hline NM033292 & CASP1 & Caspase 1 , apoptosis-related cysteine protease (interleukin $1, \beta$, convertase) & 16.2552 \\
\hline NM000700 & ANXA1 & Annexin A1 & 13.3031 \\
\hline NM001353 & AKRIC1 & $\begin{array}{l}\text { Aldo-keto reductase family } 1 \text {, member C1 (dihydrodiol dehydrogenase } 1 ; 20-\alpha(3-\alpha) \text { - } \\
\text { hydroxysteroid dehydrogenase) }\end{array}$ & 10.4311 \\
\hline NM001818 & AKRIC4 & $\begin{array}{l}\text { Aldo-keto reductase family } 1 \text {, member } \mathrm{C} 4 \text { (chlordecone reductase; } 3 \text { - } \alpha \text { hydroxysteroid } \\
\text { dehydrogenase) }\end{array}$ & 10.3319 \\
\hline NM020038 & MRP3 & ATP-binding cassette, sub-family C (CFTR/MRP), member 3 & 3.9728 \\
\hline NM016234 & ACSL5 & Fatty-acid-coenzyme A ligase, long-chain 5 & 3.7045 \\
\hline NM004572 & PKP2 & Plakophilin 2 & 3.5271 \\
\hline NM024563 & FLJ14054 & Hypothetical protein FLJ14054 & 3.4879 \\
\hline NM003054 & SLC18A2 & Solute carrier family 18 (vesicular monoamine), member 2 & 3.2583 \\
\hline NM002015 & FOXO1A & Forkhead box O1A (rhabdomyosarcoma) & 3.2449 \\
\hline NM001218 & CA12 & Carbonic anhydrase XII & 3.1765 \\
\hline NM000602 & SERPINE1 & Serine (or cysteine) proteinase inhibitor, clade E (nexin, plasminogen activator inhibitor type 1) & 3.1525 \\
\hline NM024659 & FLJ11753 & Hypothetical protein FLJ11753 & 3.0686 \\
\hline NM002539 & ODC1 & Ornithine decarboxylase 1 & 2.8646 \\
\hline AF072928 & MTMR6 & Myotubularin-related protein 6 & 2.8512 \\
\hline AK057568 & INE2 & Inactivation escape 2 & 2.8102 \\
\hline AF019226 & GOV & Glioblastoma overexpressed & 2.7968 \\
\hline BC017984 & LOC338769 & Homo sapiens, clone IMAGE: 4245930, mRNA & 2.7906 \\
\hline NM032883 & C20orf100 & Chromosome 20 open reading frame 100 & 2.7100 \\
\hline AK055962 & ARG99 & ARG99 protein & 2.6579 \\
\hline NM002097 & GTF3A & General transcription factor IIIA & 2.6531 \\
\hline NM001855 & COL15A1 & Collagen, type XV, $\alpha 1$ & 2.6529 \\
\hline NM020987 & ANK3 & Ankyrin 3, node of Ranvier (ankyrin G) & 2.6458 \\
\hline AB058718 & KIAA1815 & Hypothetical protein FLJ23309 & 2.5897 \\
\hline AF218006 & FLJ13612 & Hypothetical protein FLJ13612 & 2.5560 \\
\hline NM024696 & FLJ23058 & Hypothetical protein FLJ23058 & 2.5358 \\
\hline NM006437 & PARP4 & ADP-ribosyltransferase (NAD ${ }^{+}$; poly (ADP-ribose) polymerase)-like 1 & 2.5014 \\
\hline NM002620 & PF4V1 & Platelet factor 4 variant 1 & 2.4987 \\
\hline NM003558 & PIP5K1B & Unc-13-like (C.elegans) & 2.4719 \\
\hline NM002317 & LOX & Lysyl oxidase & 2.4123 \\
\hline ВC015417 & MGC21981 & Homo sapiens, clone MGC: 21981 IMAGE: 4396073, mRNA, complete cds & 2.4026 \\
\hline NM002061 & GCLM & Glutamate-cysteine ligase, modifier subunit & 2.3993 \\
\hline NM003039 & SLC2A5 & Solute carrier family 2 (facilitated glucose/fructose transporter), member 5 & 2.3748 \\
\hline NM033128 & SCIN & Scinderin & 2.3618 \\
\hline NM001038 & SCNN1A & Sodium channel, nonvoltage-gated $1 \alpha$ & 2.3547 \\
\hline NM004751 & GCNT3 & Glucosaminyl (N-acetyl) transferase 3 , mucin type & 2.3368 \\
\hline SK025736 & HMGCS1 & 3-Hydroxy-3-methylglutaryl-coenzyme a synthase 1 (soluble) & 2.3321 \\
\hline NM000716 & C4BPB & Complement component 4 binding protein, $\beta$ & 2.3225 \\
\hline NM005857 & ZMPSTE24 & Zinc metalloproteinase (STE24 homolog, yeast) & 2.3135 \\
\hline NM001498 & GCLC & Glutamate-cysteine ligase, catalytic subunit & 2.3049 \\
\hline NM004105 & EFEMP1 & EGF-containing fibulin-like extracellular matrix protein 1 & 2.2991 \\
\hline AB018271 & KIAA0728 & KIAA0728 protein & 2.2949 \\
\hline NM016542 & MST4 & Serine/threonine protein kinase MASK & 2.2656 \\
\hline AL050201 & C6orf162 & Hypothetical protein DKFZp586E1923 & 2.2518 \\
\hline ВС010117 & MGC13038 & Hypothetical protein MGC13038 & 2.2448 \\
\hline NM002395 & ME1 & Malic enzyme 1, NADP(+)-dependent, cytosolic & 2.2398 \\
\hline NM000908 & NPR3 & Natriuretic peptide receptor C/guanylate cyclase $\mathrm{C}$ (atrionatriuretic peptide receptor $\mathrm{C}$ ) & 2.2196 \\
\hline NM000237 & LPL & Lipoprotein lipase & 2.2196 \\
\hline NM004529 & MLLT3 & Myeloid/lymphoid or mixed-lineage leukemia (trithorax homolog, Drosophila); translocated to, 3 & 2.2057 \\
\hline AB002351 & DMN & Desmuslin & 2.1525 \\
\hline D80010 & LPIN1 & Lipin 1 & 2.1335 \\
\hline NM022358 & KCNK15 & Potassium channel, subfamily K, member 15 (TASK-5) & 2.1214 \\
\hline NM001845 & COL4A1 & Collagen, type IV, $\alpha 1$ & 2.1074 \\
\hline
\end{tabular}


Table I. Continued.

\begin{tabular}{|c|c|c|c|}
\hline Accession no. & Gene name & Gene description & Ratio \\
\hline AK000160 & MYO1B & Myosin IB & 2.0118 \\
\hline AF361486 & MUC16 & Mucin 16 & 0.4869 \\
\hline NM014279 & OLFM1 & Olfactomedin 1 & 0.4856 \\
\hline NM004494 & HDGF & Hepatoma-derived growth factor (high-mobility group protein 1-like) & 0.4652 \\
\hline AF070673 & SNN & Stannin & 0.4634 \\
\hline AL390079 & LOC58489 & Hypothetical protein from EUROIMAGE 588495 & 0.4594 \\
\hline NM001908 & CTSB & Cathepsin B & 0.4572 \\
\hline NM000480 & AMPD3 & Adenosine monophosphate deaminase (isoform E) & 0.4489 \\
\hline АВ011100 & KIAA0528 & KIAA0528 gene product & 0.4419 \\
\hline NM005239 & ETS2 & V-ets erythroblastosis virus E26 oncogene homolog 2 (avian) & 0.4393 \\
\hline AB040879 & KIAA1446 & KIAA1446 protein & 0.4371 \\
\hline AF217965 & PTGES & Prostaglandin E synthase & 0.4261 \\
\hline NM006622 & PLK2 & Serum-inducible kinase & 0.4178 \\
\hline NM002583 & PAWR & PRKC, apoptosis, WT1, regulator & 0.4095 \\
\hline NM001902 & $\mathrm{CTH}$ & Cystathionase (cystathionine $\gamma$-lyase) & 0.4061 \\
\hline NM031915 & SETDB2 & CLLL8 protein & 0.4053 \\
\hline NM001848 & COL6A1 & Collagen, type VI, $\alpha 1$ & 0.4004 \\
\hline AF022789 & USP12 & Ubiquitin specific protease 12 & 0.3945 \\
\hline AJ311123 & DKFZP434A1114 & Hypothetical gene DKFZp434A1114 & 0.3939 \\
\hline NM001089 & $\mathrm{ABCA} 3$ & ATP-binding cassette, sub-family A ( $\mathrm{ABC} 1)$, member 3 & 0.392 \\
\hline NM012168 & FBXO2 & F-box only protein 2 & 0.3916 \\
\hline NM024808 & FLJ22624 & Hypothetical protein FLJ22624 & 0.3908 \\
\hline NM024110 & CARD14 & Caspase recruitment domain protein 14 & 0.3898 \\
\hline NM021012 & $\mathrm{KCNJ} 12$ & Potassium inwardly-rectifying channel, subfamily $\mathrm{J}$, member 12 & 0.3878 \\
\hline NM000362 & TIMP3 & Tissue inhibitor of metalloproteinase 3 (sorsby fundus dystrophy, pseudoinflammatory) & 0.3869 \\
\hline NM031412 & GABARAPL1 & GABA(A) receptor-associated protein like 1 & 0.3865 \\
\hline AK024478 & FLJ11305 & Hypothetical protein FLJ11305 & 0.3857 \\
\hline NM014116 & MED4 & HSPC126 protein & 0.3818 \\
\hline NM002414 & CD99 & Antigen identified by monoclonal antibodies $12 \mathrm{E} 7, \mathrm{~F} 21$ and $\mathrm{O} 13$ & 0.3745 \\
\hline NM017720 & FLJ20234 & Hypothetical protein FLJ20234 & 0.3723 \\
\hline NM003069 & SMARCA1 & $\begin{array}{l}\text { SWI/SNF related, matrix associated, actin dependent regulator of chromatin, } \\
\text { subfamily a, member } 1\end{array}$ & 0.3695 \\
\hline NM017905 & C13orf11 & Hypothetical protein FLJ20623 & 0.3693 \\
\hline NM002961 & S100A4 & $\begin{array}{l}\text { S100 calcium binding protein } \mathrm{A} 4 \text { (calcium protein, calvasculin, metastasin, } \\
\text { murine placental homolog) }\end{array}$ & 0.3636 \\
\hline AK056841 & KCNRG & Homo sapiens cDNA FLJ32279 fis, clone PROST2000165 & 0.3595 \\
\hline NM007150 & ZNF185 & Zinc finger protein 185 (LIM domain) & 0.3579 \\
\hline NM018214 & LANO & LAP (leucine-rich repeats and PDZ) and no PDZ protein & 0.3552 \\
\hline D83076 & KIAA0239 & KIAA0239 protein & 0.3538 \\
\hline NM006186 & NR4A2 & Nuclear receptor subfamily 4 , group A, member 2 & 0.3534 \\
\hline NM025206 & FER1L4 & Fer-1-like 4 (C. elegans) & 0.3529 \\
\hline NM003652 & $\mathrm{CPZ}$ & Carboxypeptidase Z & 0.3527 \\
\hline AK027071 & $\mathrm{TSC} 22$ & Transforming growth factor $\beta$-stimulated protein TSC- 22 & 0.3522 \\
\hline U67784 & $\mathrm{RDC} 1$ & G protein-coupled receptor & 0.3517 \\
\hline NM002747 & MAPK4 & Mitogen-activated protein kinase 4 & 0.3506 \\
\hline NM032401 & TMPRSS3 & Transmembrane protease, serine 3 & 0.3493 \\
\hline NM018584 & PRO1489 & Hypothetical protein PRO1489 & 0.3461 \\
\hline NM052811 & RFP2 & Ret finger protein 2 & 0.3448 \\
\hline NM001200 & BMP2 & Bone morphogenetic protein 2 & 0.3363 \\
\hline NM000213 & ITGB4 & Integrin, $\beta 4$ & 0.3350 \\
\hline NM014448 & ARHGEF16 & Rho guanine exchange factor (GEF) 16 & 0.3322 \\
\hline NM019045 & WDR44 & Similar to rab11-binding protein & 0.3294 \\
\hline NM000683 & ADRA2C & Adrenergic, $\alpha-2 \mathrm{C}-$, receptor & 0.3269 \\
\hline NM004221 & NK4 & Natural killer cell transcript 4 & 0.3242 \\
\hline NM012331 & MSRA & Methionine sulfoxide reductase A & 0.3164 \\
\hline NM003186 & TAGLN & Transgelin & 0.3045 \\
\hline
\end{tabular}


Table I. Continued.

\begin{tabular}{|c|c|c|c|}
\hline Accession no. & Gene name & Gene description & Ratio \\
\hline NM001632 & ALPP & Alkaline phosphatase, placental (regan isozyme) & 0.2972 \\
\hline NM005245 & FAT & FAT tumor suppressor homolog 1 (Drosophila) & 0.2970 \\
\hline D25304 & ARHGEF6 & Rac/Cdc42 guanine nucleotide exchange factor (GEF) 6 & 0.2942 \\
\hline $\mathrm{AB} 021124$ & CHST2 & Carbohydrate (N-acetylglucosamine-6-O) sulfotransferase 2 & 0.2905 \\
\hline NM001381 & DOK1 & Docking protein $1,62 \mathrm{kD}$ (downstream of tyrosine kinase 1 ) & 0.2837 \\
\hline NM003820 & TNFRSF14 & Tumor necrosis factor receptor superfamily, member 14 (herpesvirus entry mediator) & 0.2786 \\
\hline NM052947 & ALPK2 & Heart $\alpha$-kinase & 0.2784 \\
\hline NM018306 & FLJ11036 & Hypothetical protein FLJ11036 & 0.2778 \\
\hline AF323540 & APOL1 & Apolipoprotein L, 1 & 0.2770 \\
\hline NM016602 & CCR10 & G protein-coupled receptor 2 & 0.2754 \\
\hline AF018081 & COL18A1 & Collagen, type XVIII, $\alpha 1$ & 0.2662 \\
\hline NM018478 & C20orf35 & Chromosome 20 open reading frame 35 & 0.2579 \\
\hline NM002193 & INHBB & Inhibin, $\beta \mathrm{B}$ (activin $\mathrm{AB} \beta$ polypeptide) & 0.2430 \\
\hline NM000273 & GPR143 & Ocular albinism 1 (Nettleship-Falls) & 0.2383 \\
\hline AF217974 & DKFZP434K1772 & Hyothetical protein & 0.2307 \\
\hline NM000422 & KRT17 & Keratin 17 & 0.2246 \\
\hline AK001520 & LOC153218 & Homo sapiens cDNA FLJ10658 fis, clone NT2RP2006052 & 0.2089 \\
\hline AL137493 & DKFZP434B1231 & Homo sapiens mRNA; cDNA DKFZp434B1231 (from clone DKFZp434B1231); partial cds & 0.2074 \\
\hline NM007361 & NID2 & Nidogen 2 & 0.1986 \\
\hline NM003022 & SH3BGRL & SH3 domain binding glutamic acid-rich protein like & 0.1773 \\
\hline NM003645 & SLC27A2 & Fatty-acid-coenzyme a ligase, very long-chain 1 & 0.1668 \\
\hline BC011762 & PIR121 & Cytoplasmic FMRP interacting protein 2 & 0.1580 \\
\hline NM002354 & TACSTD1 & Tumor-associated calcium signal transducer 1 & 0.1109 \\
\hline NM024829 & FLJ22662 & Hypothetical protein FLJ22662 & 0.1102 \\
\hline АВ033073 & KIAA1247 & Similar to glucosamine-6-sulfatases & 0.0930 \\
\hline NM020373 & TEME16B & Chromosome 12 open reading frame 3 & 0.0857 \\
\hline NM031311 & CPVL & Carboxypeptidase, vitellogenic-like & 0.0847 \\
\hline NM006169 & NNMT & Nicotinamide N-methyltransferase & 0.0819 \\
\hline AL050125 & LOC283824 & Homo sapiens mRNA; cDNA DKFZp586F071 (from clone DKFZp586F071) & 0.0808 \\
\hline NM000735 & CGA & Glycoprotein hormones, $\alpha$ polypeptide & 0.0781 \\
\hline NM022368 & PJA1 & Praja 1 & 0.0714 \\
\hline NM052966 & C1orf24 & Chromosome 1 open reading frame 24 & 0.0549 \\
\hline ВC012337 & FLJ22761 & Hypothetical protein FLJ22761 & 0.0504 \\
\hline NM002639 & SERPINB5 & Serine (or cysteine) proteinase inhibitor, clade B (ovalbumin), member 5 & 0.0306 \\
\hline NM007015 & LECT1 & Chondromodulin I precursor & 0.0093 \\
\hline
\end{tabular}

magnification. The slides were evaluated by two experienced pathologists and a consensus was reached. Positive expression for Bcl-2 or MRP3 was defined as $>15 \%$ staining intensity of tumor cells. The staining of Bcl-2 was mainly localized in the cytoplasm and the staining of MRP3 was localized in the cytoplasm or membrane. Immunohistochemical staining for the Bcl-2 and MRP3 antigen was performed according to the manufacturer's instructions using the standard streptavidinperoxidase biotin technique with an SP kit (Zhongshan Co., Beijing, China).

Statistical analysis. The SAS statistical software version 9.1 was used for all calculations and statistical analyses. All results were expressed as means \pm SD. Statistical significance between treated and untreated groups and analysis of mRNA expression were determined by a Student's t-test. The Dunnett's test was used for evaluation of the inhibitory activity on tumor growth between treated and untreated groups. To assess immunohistochemical data, ANOVA was performed for comparisons between the two groups, Bonferroni's correction was used to compare the multiple comparisons of rate, and correlations between the levels of $\mathrm{Bcl}-2$ and MRP3 were determined using Pearson's correlation analysis. A two-tailed P-value $<0.05$ was considered to be statistically significant.

\section{Results}

Differential gene expression in Anip973 and Anip973/NVB cells by microarray analysis. Expression profiles established from Anip973 and Anip973/NVB cells were based on mean values from triplicate hybridizations. The complete list of these 21571 gene probes was provided as the complete microarray gene expression. One-hundred and forty-three genes were identified as differentially expressed between Anip973 and Anip973/NVB cells. Of these, 55 genes were up-regulated and 88 genes were down-regulated in Anip973/NVB cells 


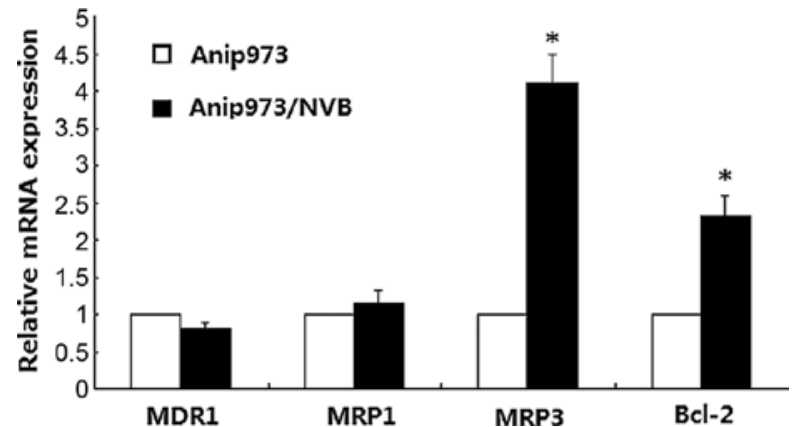

Figure 1. Relative mRNA expression of MDR1, MRP1, MRP3 and Bcl-2 in Anip973 and Anip973/NVB cells. mRNA expression of MDR1 and MRP1 was similar in Anip973/NVB cells relative to those in Anip973 cells. mRNA expression of MRP3 and Bcl-2 was up-regulated in Anip973/NVB cells relative to those in Anip973 cells ( $\mathrm{P}<0.05)$.

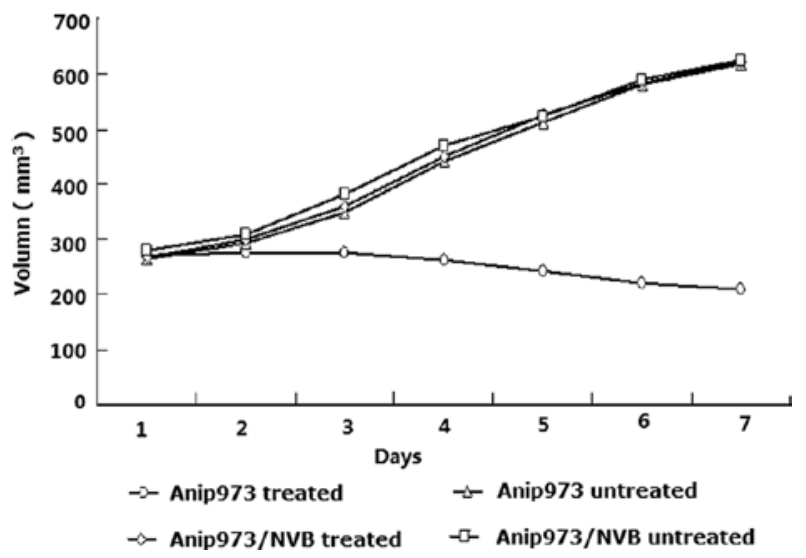

Figure 2. Tumor growth curves of Anip973 cell and Anip973/NVB cell xenografts. After treated by vinorelbine, Anip973 xenografts grew slowly and vinorelbine inhibited the growth of Anip973 xenografts $(\mathrm{P}<0.001)$. However, growth of Anip973/NVB xenografts treated by vinorelbine was similar with the 2 untreated groups.

compared to Anip973 cells (Table I). The functions of 143 differently expression genes covered the following: transport, transcription, regulation, signaling, response to stress, metabolism, development, cell organization and biogenesis, cell motility, differentiation, cell cycle, cell adhesion and apoptosis.

qRT-PCR validation of selected genes related to drug-resistance. Four genes known to be related to drug-resistance in cancer cells were selected for validation of the microarray data by qRT-PCR: MDR1, MRP1, MRP3 and Bcl-2 (Fig. 1). Results of qRT-PCR showed that the mRNA levels of MDR1 and MRP1 were similar in the two cell lines ( $\mathrm{P}>0.05)$. On the other hand, the mRNA levels of MRP3 were higher in Anip973/NVB cells compared with Anip973 cells $(\mathrm{P}<0.05)$, which confirmed the microarray data. The mRNA level of $\mathrm{Bcl}-2$ was higher in the Anip973/NVB cells compared with Anip973 cells $(\mathrm{P}<0.05)$ as determined by qRT-PCR, although there was no difference in Bcl-2 expression by microarray analysis.

Vinorelbine inhibits xenografts of Anip973 growth but does not affect xenografts of Anip973/NVB growth. Tumor growth curves are shown in Fig. 2. Two days after treatment, tumor growth in the Anip973-treated group became slow, and it was

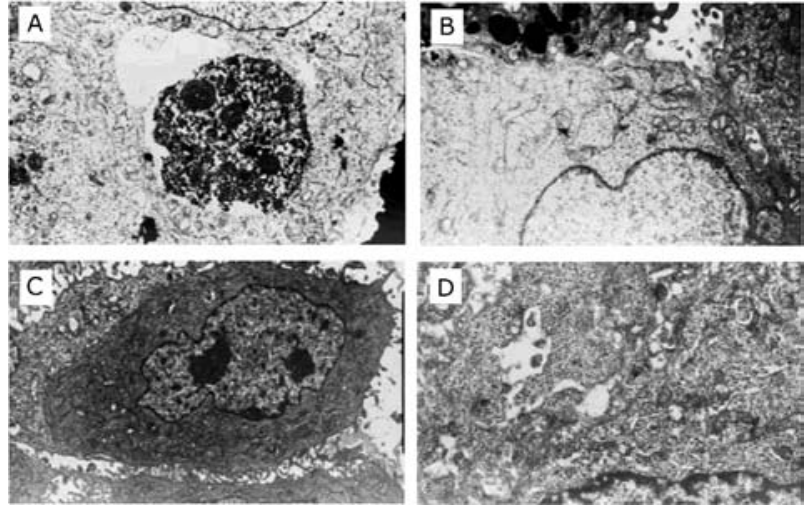

Figure 3. Morphological changes in apoptosis observed in Anip973 and Anip973/NVB xenografts after treatment with vinorelbine by TEM (x10,000). Anip973 cells (A and B) presented apoptosis characteristics, such as cell shrinkage, loss of microvilli and nucleolus, chromatin condensation and formation of apoptotic bodies. No apoptotic changes were observed in Anip973/NVB cells (C and D).

significantly different from the other groups. Seven days after treatment, tumor growth inhibition was obviously observed in the Anip973-treated group. The tumor growth inhibition rates in the Anip973-treated group and the Anip973/NVB-treated group were $60.00 \%$ and $4.65 \%$ respectively, significantly different between two treated groups $(\mathrm{P}<0.001)$. However, there was no significant difference between the tumor growth in the Anip973-untreated group, the Anip973/NVB-untreated group and the Anip973/NVB-treated group $(\mathrm{P}=0.358)$.

Vinorelbine induces apoptosis in Anip973 cells, but not in Anip973/NVB cells. Ultrastructural changes were observed by TEM (Fig. 3). Results showed that cells of the Anip973-treated group underwent serial changes, such as cell shrinkage, loss of microvilli and nucleolus, chromatin condensation and formation of apoptotic bodies, which were characteristic of apoptosis in cells. Cytological observations of the Anip973/ NVB treated group showed no apoptotic changes and the presence of microvilli and nucleoli and of a large number of well-developed organelles, including Golgi complexes.

Expression of $\mathrm{Bcl}-2$ and MRP3 in tumor xenografts. Higher expression rates of $\mathrm{Bcl}-2$ and MRP3 were observed in Anip973/ NVB xenograft cells compared with Anip973 xenograft cells $(\mathrm{P}<0.001)$ (Fig. 4). The expression rate of Bcl-2 in the Anip973treated group, Anip973-untreated group, Anip973/NVB-treated group and Anip973/NVB-untreated group was 47, 48, 74 and $72 \%$, respectively. Moreover, the positive expression rate of MRP3 in the Anip973-treated group, Anip973-untreated group, Anip973/NVB-treated group and Anip973/NVB-untreated group was $53,52,79$ and $76 \%$, respectively. No correlation was noted between $\mathrm{Bcl}-2$ and MRP3 $(\mathrm{P}=0.091)$.

\section{Discussion}

As an important anticancer drug, NVB is widely used as chemotherapeutic agent in the treatment of a variety of malignancies, including NSCLC (9), metastatic breast cancer (10), ovarian cancer (11), Hodgkin's disease (12), small cell lung cancer (13), and esophageal carcinoma (14). However, because 

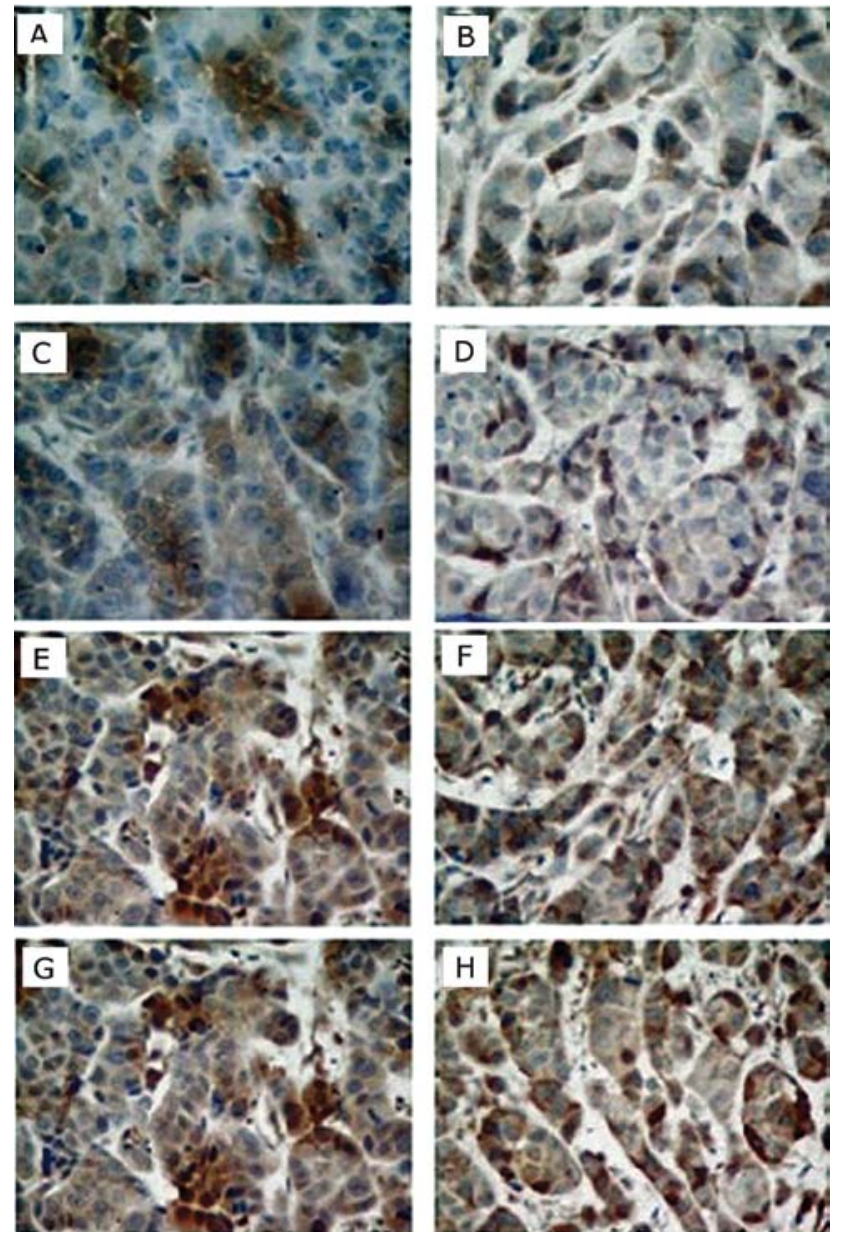

Figure 4. The expression of Bcl-2 (A, C, E and G) and MRP3 (B, D, F and H) in Anip973 and Anip973/NVB xenografts by immunohistochemistry (x400). The expression of Bcl-2 in Anip973/NVB-treated (E) and untreated xenografts (G) was higher than in the Anip973-treated (A) and untreated xenografts (C) and the expression of MRP3 in the Anip973/NVB-treated (F) and untreated xenografts (H) was higher than in the Anip973-treated (B) and untreated xenografts (D) both treated by vinorelbine and saline.

of acquired drug resistance, NVB is not effective after several chemotherapeutic cycles. To study the possible mechanisms of multidrug resistance induced by NVB in lung adenocarcinoma, we established the resistant cell line Anip973/NVB induced by NVB from the parental cell line Anip973, the characteristics of which were confirmed in a previous study (8). Establishment of a multidrug resistance cell line of lung adenocarcinoma was essential and significant for our present study.

In the present study, we first investigated the gene expression variance between the Anip973/NVB and Anip973 cell lines by DNA microarray analysis. This is the first report describing variations in gene expression and genomic alterations of multidrug resistance by NVB in lung adenocarcinomas using microarray technologies. We found that several genes were differentially expressed in the two cell lines, involving cell transport, transcription, regulation, signaling, response to stress, metabolism, development, cell organization and biogenesis, cell motility, differentiation, cell cycle, cell adhesion and apoptosis. Of these genes, the functions of some are still not validated and some genes have multiple functions, therefore selecting genes for further research was difficult. Although some genes were differently expressed significantly between two cells, such as SCAP1, CASP1, ANXA1, AKRIC1, AKRIC4, LECT1, SERPINB5, FLJ22761, C1orf24, PJA1, CGA, LOC283824, NNMT, CPVL, TEME16B, KIAA1247, we decided to study them in the future.

Of the 143 differentially expressed genes, up-regulation of the expression of MRP3 being a drug resistance gene in Anip973/NVB cells was of interest. In addition to MRP3, we selected other classic genes related to drug resistance to confirm the results of our microarray analysis. Results of qRT-PCR showed that the expression of MDR1, MRP1 and MRP3 were in agreement to those of the microarray analysis. However, the mRNA level of Bcl-2 was higher in Anip973/ NVB cells compared with Anip973 cells, although no difference was observed in the microarray data between the two cells. We searched the microarray data and found that the ratios of Bcl-2 was 1.7402, 7.5587, 1.7379 respectively in three experiments. As mentioned in our methods, in order to avoid false positive results, only when the ratio of one gene was $>2$ in all three experiments, the gene was considered to show a significant differential expression between the two cell lines. It is for this reason that $\mathrm{Bcl}-2$ was excluded from the differential genes identified by microarray analysis. Nevertheless, we identified a trend for Bcl-2 up-regulation in Anip973/NVB cells compared with Anip973 cells by both methods.

To examine the response of Anip973 and Anip973/NVB cells to NVB in vivo, we established mice xenograft models. We found that Anip973/NVB xenografts resisted NVB therapy, which reconfirmed the characteristics of Anip973/ NVB cells. We did not observe cell apoptosis by TEM in Anip973/NVB xenografts after NVB therapy, suggesting that anti-apoptosis may be one of the drug-resistance mechanisms. Furthermore, we performed immunohistochemistry to observe the expression of Bcl-2 and MRP3 protein. As expected, higher expression of Bcl-2 and MRP3 were demostrated in Anip973/ NVB xenografts cells compared with Anip973 xenograft cells, that further validated our findings of gene variation.

P-glycoprotein (MDR1) and multidrug resistance protein 1 (MRP1) play an important role in the extrusion of drugs from cancer cells and their overexpression can be a cause of failure of anticancer drugs in numerous cancers $(15,16)$. Results both from DNA microarray analysis and qRT-PCR showed that MDR1 and MRP1 were not responsible for resistance to NVB in the present study.

Belonging to the ATP-binding cassette (ABC) multidrug transporters that constitute the MRP family, MRP3 is an organic anion transporter capable of transporting anticancer agents, and it can actively efflux lipophilic anions and hydrophobic compounds and contribute to multidrug resistance in cancers $(17,18)$. Originally, investigators reported that MRP3 mRNA levels were strongly correlated with resistance to doxorubicin, vincristine, etoposide and cisplatin in lung cancer cells $(19,20)$. Subsequently, MRP3 was found that it contributed to 5-fluorouracil resistance in pancreatic carcinoma cells (21). Huang et al (22) found that MRP3 contributed to vincristine resistance in human cancer cells, however, this was cell-selective and was not necessarily dependent on pregnane X receptor-mediated effects. Furthermore, MRP3 also contributed to adriamycin resistance in human hepatocellular 
carcinoma cells and tetramethylpyrazine could effectively reverse the multi-drug resistance (23). The results of our study showed that mRNA and protein expression of MRP3 were higher in Anip973/NVB cells than in Anip973 cells, indicating that MRP3 may participate in NVB resistance in lung adenocarcinoma. According to NVB being a lipophilic and hydrophobic drug, we speculated that MRP3 may efflux NVB from cells and contribute to multidrug resistance in lung adenocarcinoma.

Although proteins that interfere with either drug accumulation or efflux contribute to drug resistance, other factors also play an important role. One major mechanism of drug resistance in cancer cells is the defective apoptosis pathway $(24,25)$. In our study, we also found that up-regulation of Bcl-2 may be involved in multi-drug resistance induced by NVB in lung adenocarcinoma. It is known that Bcl-2 is an important anti-apoptotic protein that can regulate cancer cell apoptosis and prolong cell survival (26). In addition, Bcl-2 has been known to confer resistance to chemotherapeutic agents in a variety of human cancers (27). Studies in acute myeloid leukemia (AML) have revealed that high levels of Bcl-2 were associated with resistance to chemotherapy and antisense oligodeoxynucleotides targeting Bcl-2 mRNA could increase sensitivity of AML cells to arabinocytosine in vitro $(28,29)$. Researchers found that overexpression of Bcl-2 was associated with resistance to chemotherapy-induced apoptosis in pancreatic cancer and the ERK/Bcl-2 pathway may confer resistance of pancreatic cancer to anoikis $(30,31)$. As reported, we consider that $\mathrm{Bcl}-2$ confers drug resistance by preventing NVB-induced cell death in lung adenocarcinoma in the present study, that was confirmed by results observed through TEM.

In summary, we identified a set of genes that were responsible for multidrug resistance induced by NVB in lung adenocarcinoma. Among them, contribution of MRP3 and Bcl-2 were confirmed in vitro and in vivo. However, a great number of factors are documented to be associated with drug resistance in human cancer. In future studies we plan to investigate other differentially expressed genes identified using DNA microarray and further study the mechanism of NVB drug resistance in lung adenocarcinoma.

\section{Acknowledgements}

We thank Dr Sun Xilin for excellent advice on preparing the manuscript. This study was supported by the Scientific and Technological Innovative Project of Harbin (no. 2006RFXXS037).

\section{References}

1. Marty M, Extra JM, Espie M, et al: Advances in vinca-alkaloids: Navelbine. Nouv Rev Fr Hematol 31: 77-84, 1989.

2. Rahmani-Jourdheuil D, Coloma F, Placidi M, et al: Human hepatic uptake of two vinca alkaloids: navelbine and vincristine. J Pharm Sci 83: 468-471, 1994.

3. Vokes EE, Rosenberg RK, Jahanzeb M, et al: Multicenter phase II study of weekly oral vinorelbine for stage IV non-small-cell lung cancer. J Clin Oncol 13: 637-644, 1995.

4. Gebbia V, Caruso M, Valenza R, et al: Vinorelbine plus cisplatinum for the treatment of stage IIIB and IV non small cell lung carcinoma. Anticancer Res 14: 1247-1249, 1994.
5. Green MR: New directions for chemotherapy in non-small-cell lung cancer. Chest 103 (Suppl 4): S370-S372, 1993.

6. Muller M, Meijer C, Zaman GJ, et al: Overexpression of the gene encoding the multidrug resistance-associated protein results in increased ATP-dependent glutathione S-conjugate transport. Proc Natl Acad Sci USA 91: 13033-13037, 1994.

7. Hipfner DR, Deeley RG and Cole SP: Structural, mechanistic and clinical aspects of MRP1. Biochim Biophys Acta 1461: 359-376, 1999.

8. Chen GY, Yang ZY, Hong X, et al: Establishment of a multi drugresistant human lung adenocarcinoma cell line and biological characteristics. Zhonghua Yi Xue Za Zhi 87: 924-926, 2007.

9. Rinaldi M, Cauchi C and Gridelli C: First line chemotherapy in advanced or metastatic NSCLC. Ann Oncol 17: 64-67, 2006.

10. Domenech GH and Vogel CL: A review of vinorelbine in the treatment of breast cancer. Clin Breast Cancer 2: 113-128, 2001.

11. Harries M and Kaye SB: Recent advances in the treatment of epithelial ovarian cancer. Expert Opin Investig Drugs 10: 1715-1724, 2001.

12. Devizzi L, Santoro A, Bonfante V, et al: Vinorelbine: a new promising drug in Hodgkin's disease. Leuk Lymphoma 22: 409-414, 1996.

13. Weinmann M, Jeremic B, Bamberg M, et al: Treatment of lung cancer in elderly part II: small cell lung cancer. Lung Cancer 40: 1-16, 2003.

14. Bidoli P, Aglione S, Toffolatti L, et al: New strategies in the treatment of esophageal cancer. Minerva Chir 57: 717-731, 2002.

15. He SM, Li R, Kanwar JR, et al: Structural and functional properties of human multidrug resistance protein 1 (MRP1/ABCC1). Curr Med Chem 18: 439-481, 2011.

16. Li X, Li JP, Yuan HY, et al: Recent advances in P-glycoproteinmediated multidrug resistance reversal mechanisms. Methods Find Exp Clin Pharmacol 29: 607-617, 2007.

17. Borst $\mathrm{P}$ and Elferink RO: Mammalian ABC transporters in health and disease. Annu Rev Biochem 71: 537-592, 2002.

18. Kruh GD and Belinsky MG: The MRP family of drug efflux pumps. Oncogene 22: 7537-7552, 2003.

19. Young LC, Campling BG, Voskoglou-Nomikos T, et al: Expression of multidrug resistance protein-related genes in lung cancer: correlation with drug response. Clin Cancer Res 5: 673-680, 1999.

20. Oguri T, Isobe T, Fujitaka K, et al: Association between expression of the MRP3 gene and exposure to platinum drugs in lung cancer. Int J Cancer 93: 584-589, 2001.

21. Hagmann W, Jesnowski R, Faissner R, et al: ATP-binding cassette $\mathrm{C}$ transporters in human pancreatic carcinoma cell lines. Up-regulation in 5-fluorouracil-resistant cells. Pancreatology 9: 136-144, 2009.

22. Huang R, Murry DJ, Kolwankar D, et al: Vincristine transcriptional regulation of efflux drug transporters in carcinoma cell lines. Biochem Pharmacol 71: 1695-1704, 2006.

23. Wang XB, Wang SS, Zhang QF, et al: Inhibition of tetramethylpyrazine on P-gp, MRP2, MRP3 and MRP5 in multidrug resistant human hepatocellular carcinoma cells. Oncol Rep 23: 211-215, 2010.

24. Johnstone RW, Ruefli AA and Lowe SW: Apoptosis: a link between cancer genetics and chemotherapy. Cell 108: 153-164, 2002.

25. Reed JC: Dysregulation of apoptosis in cancer. J Clin Oncol 17: 2941-2953, 1999.

26. Zhou F, Yang Y and Xing D: Bcl-2 and Bcl-xL play important roles in the crosstalk between autophagy and apoptosis. FEBS J 278: 403-413, 2011.

27. Reed JC: Bcl-2-family proteins and hematologic malignancies: history and future prospects. Blood 111: 3322-3330, 2008.

28. Tóthová E, Fricova M, Stecová N, et al: High expression of Bcl-2 protein in acute myeloid leukemia cells is associated with poor response to chemotherapy. Neoplasma 49: 141-144, 2002.

29. Campos L, Sabido O, Rouault JP, et al: Effects of BCL-2 antisense oligodeoxynucleotides on in vitro proliferation and survival of normal marrow progenitors and leukemic cells. Blood 84: 595-600, 1994.

30. Bold RJ, Chandra J and McConkey DJ: Gemcitabine-induced programmed cell death (apoptosis) of human pancreatic carcinoma is determined by Bcl-2 content. Ann Surg Oncol 6: 279-285, 1999.

31. Galante JM, Mortenson MM, Bowles TL, et al: ERK/BCL-2 pathway in the resistance of pancreatic cancer to anoikis. J Surg Res 152: 18-25, 2009. 